\title{
The psychiatric inpatient physical health assessment sheet (PIPHAS): a useful tool to improve the speed, efficiency, and documentation of physical examination in new psychiatric inpatients
}

\author{
Alexander Pettipher, Richard Ovens
}

Prospect Park Hospital, UK

\begin{abstract}
There is increased morbidity and mortality among patients suffering from mental illness. This is believed to be multi-factorial. Poor access to healthcare, the stigma of mental illness, reduced clinic attendance, lifestyle factors, and side effects of medications are cited as possible contributing factors. It is therefore vital to perform a physical examination to identify previously undiagnosed conditions during the admission of a psychiatric inpatient. The Royal College of Psychiatrists recommends that all patients admitted to a psychiatric hospital should receive a full physical examination on admission, or within twenty-four hours of admission.
\end{abstract}

A snapshot audit was carried out at Prospect Park Hospital in Reading, which highlighted that The Royal College of Psychiatrist's recommendation, along with Trust guidelines regarding physical examination were not being met, with only 78 out of 111 patients (70.3\%) undergoing an examination during their admission. In addition to this, examinations were often poorly documented and not covering all examination domains. A psychiatric inpatient physical health assessment sheet (PIPHAS) was designed and introduced, providing a quick and standardised approach to the documentation of a physical examination. After the intervention was put into practice, its impact was assessed by performing a retrospective review of the admission clerking notes of the next 100 admissions to Prospect Park Hospital.

Following the introduction of the PIPHAS form there was an increase in the number of patients undergoing physical examination on admission to hospital ( 75 out of 100 patients, $75 \%$ ). There was also an increase in the thorough documentation of all examination domains (e.g. respiratory examination) for patients that had a completed PIPHAS form scanned within their medical records.

This quality improvement project demonstrates that the PIPHAS form is a useful tool to improve the speed, efficiency, and documentation of a thorough physical examination in new psychiatric inpatients.

\section{Problem}

When observing psychiatric inpatients, the identification and treatment of physical health complaints is often deemed less important, or less of a priority, than addressing their mental health needs. The aim of this project is ultimately to improve the recognition of physical health complaints in psychiatric inpatients at Prospect Park Hospital, Berkshire, United Kingdom.

Specifically, we wanted to increase the number of adult general psychiatric inpatients who receive a prompt and thorough physical examination on admission to Prospect Park Hospital and its' documentation within their medical records.

\section{Background}

The psychiatric inpatient physical health audit (PIPHA) focussed on the physical examination of acute general adult psychiatric inpatients at Prospect Park Hospital in Reading, Berkshire. Physical assessment is vital in order to aid the exclusion of an organic pathology as the aetiology of their mental illness. Furthermore, patients with mental illness are known to have a higher morbidity and mortality level,[1,2] with the WHO ranking acute psychosis as the third most disabling condition.[3] A review of the literature suggests the reasons behind the increased levels of morbidity and mortality are thought to be largely multifactorial.

The level of morbidity and mortality from cardiovascular disease, respiratory disease, and malignancy have all been found to be significantly higher in those with mental illness.[4,5] Access to health care has been cited as one of the reasons behind this, with the separation of mental and physical health services alongside stigma associated with mental illness as potential reasons for the increased level. Further, adherence to treatment has been cited as another potential cause, with patients with mental illness less likely to continue with treatment and attend follow up clinics.[2]

In an extensive review by De Hert et al (2011), both lifestyle and medications for the treatment of mental illness were found to be significant contributors to increased morbidity.[1] The treatment options used in the management of mental illness are well known. However, in addition to their benefits many are known to have associated side effects that can be detrimental to the overall health of the patient. For example, many anti-psychotic medications can lead to increased weight gain and the development of diabetes mellitus. Other confounders, including increased levels of smoking, substance abuse, alcohol, lack of exercise, and obesity in patients 
with mental illness are thought to further contribute.[2]

In light of this, it is clearly imperative that psychiatric inpatients undergo a thorough physical examination in order to screen them for previously undetected co-morbidities. The Royal College of Psychiatrists recognise the reduced life expectancy of those with mental health illness and have created a report and clinical guidance (listed on their website) to raise awareness of the issue.[6]

The Royal College of Psychiatrists therefore recommend that all patients admitted to a psychiatric hospital receive a full physical examination on admission, or within twenty-four hours of admission to hospital and investigations carried out promptly with clear documentation.[7] Prospect Park Hospital have drafted guidelines regarding physical health monitoring of psychiatric inpatients and these were used to develop our six audit standards.

[See attached audit standards - "An image to show our six audit standards, our intended compliance rate, and clinical exceptions."]

[See attached data collection website - "A screenshot of our online data collection portal showing our audit proforma."]

See supplementary file: ds4596.jpg - "An image to show our audit standards and data collection proforma"

\section{Baseline measurement}

A snapshot audit was carried out on a random weekday morning of all psychiatric inpatients at Prospect Park Hospital. A mid-week day was chosen to allow time for patients admitted over the previous weekend by the "on call" team, that may or may not have had a physical examination on admission. This was to be reviewed by their "day team" and allow time for them to carry out the examination. A retrospective review of inpatient medical notes was carried out, looking in particular at their admission clerking documentation, which was used to complete an audit proforma. The audit proforma was designed in-line with the trust guidelines and recommendations for inpatient physical health assessment. The notes of all 111 psychiatric inpatients on that day were reviewed, and secure online data collection was carried out using a MySQL database.

An overview of the initial audit results were as follows:

A total of 111 patients were included in the pre-intervention data collection, of which 64 were males (57.7\%). The mean age of participants was 48.4 years (with a standard deviation of 22.3 years). It appeared that the vast majority of psychiatric inpatients at Prospect Park were admitted "out-of-hours" by the on call team (91 out of 111 patients, $82 \%)$. Although a reasonable proportion of patients (78 out of 111 patients, $70.3 \%$ ) underwent a physical examination on admission to Prospect Park Hospital, there was still significant room for improvement.

Only seven of the patients that had not been examined on admission (seven out of 33 patients, $21.2 \%$ ) underwent a physical examination within 48 hours. This Suggests that the day teams were poor at identifying new admissions that were yet to have a physical examination. This meant that almost a quarter of all patients received no physical examination (26 out of 111 patients, 23.4\%).

It was of concern that no female patients over 65 years of age were offered or received a screening breast examination in-line with trust guidelines (zero out of 19 patients, $0 \%$ ). On the whole, physical examination domains were often missed out, not thoroughly examined, or poorly documented leaving a significant room for improvement. A total of 85 of the 111 patients $(76.6 \%)$ received a physical examination during their inpatient stay.

Only 34 (out of 85 patients, $40 \%$ ) had a documented level of consciousness and 46 (out of 85 patients, $54.1 \%$ ) had their temperature recorded. Thirty two (out of 85 patients, $37.6 \%$ ) had their body mass index (BMI) recorded in full; with 19 (out of 85 patients, $22.4 \%$ ) having it only partly recorded, eg height or weight. The most thoroughly documented examination domain was performing a cardiovascular examination (71 out of 85 patients, $83.5 \%$ ), closely followed by a respiratory examination (70 out of 85 patients, $82.4 \%$ ). An abdominal examination was performed on just over three-quarters of patients examined (66 out of 85 patients, $77.6 \%)$. The documentation of performing peripheral and central nervous system examinations was much less frequent, with 56 out of 85 patients $(65.9 \%)$ and 51 out of 85 patients $(60.0 \%)$ respectively. Reassuringly, most of the patients underwent phlebotomy during their inpatient stay (90 out of 111 patients, $81.1 \%)$.

See supplementary file: ds4379.xls - "Appendix - A spreadsheet to show the data obtained from the Baseline Measurement."

\section{Design}

After carrying out a focus group with other junior doctors it was decided that a "psychiatric inpatient physical health assessment sheet" (PIPHAS) would be a good way of addressing the recognised problems. The PIPHAS form is a single sided A4 proforma that facilitates the documentation of a clear and concise physical examination in a quick and easy fashion. This would serve as a prompt to remind doctors of the domains of a "thorough" physical examination, it provides a standardised and easy way to clearly document the findings of the examination (in line with Trust requirements/guidelines), and most importantly it provides an effective handover from the on call team to the day team as to which patients still require an examination or blood test, ie which patients did not undergo a physical examination on admission.

The form was designed to only fill one side of A4 paper in order to improve doctor completion rates and so that it used less resources. If the PIPHAS form was a successful intervention, then the ultimate aim is that it becomes part of the mandatory trust admission pack (along with a drug chart, VTE assessment form, medicines reconciliation form, and doctors clerking checklist). After this, all of the above forms are scanned into the patients electronic notes.

[ See "PIPHAS PDSA Cycles \& Final PIPHAS Form \& Trust 
Admission Packs Image" - to see the final PIPHAS form].

\section{Strategy}

PDSA cycle 1 (pre-intervention): Involved obtaining qualitative data from colleagues to identify if further information needed to be added to the PIPHAS form, whether or not it used a logical structure, whether or not any information requested was not necessary, and also people's overall perceptions of the form. Poor feedback rates were obtained, but the feedback received suggested sensible amendments. The PIPHAS form was amended; a blood test and ECG section was added. The form was re-structured and aesthetics altered to make it a more easy to use structure.

PDSA cycle 2 (mid-intervention): Involved obtaining quantitative data to see if it was able to be used easily in clinical practice, testing out the PIPHAS form by using it for five of the next inpatient admission clerkings. This was done in an anonymous fashion, and it was not uploaded into the patients medical records. For patients with no significant abnormalities on examination, it was surprising how long it took to complete the PIPHAS form, so changes were implemented. The PIPHAS form was amended, adding in a series of "negative" examination findings (allowing a quicker and easier way of documenting a normal examination, but allowing it to be documented in a thorough way) rather than simply writing "N.A.D" (nothing abnormal detected). For example, in the respiratory examination domain "chest clear" or "equal air entry" were added as a series of tick-boxes. All five of these patients received an examination and phlebotomy on admission along with a thorough examination, and this was documented in its' entirety as per trust guidance. However, this initial data could be skewed due to the authors completing the PIPHAS form that they were already familiar with.

Having made the above changes, the PIPHAS form was then implemented, ready for the study PDSA cycle (post-intervention). The PIPHAS form was integrated into the Trust's "new admission pack" for patients. One hundred and fifty packs were photocopied and compiled (including the new PIPHAS form, drug chart, VTE assessment form, medicines reconciliation form, blood test request form and doctors clerking checklist) and then distributed to the wards. These packs were pre-prepared in order to improve compliance with the intervention. Since the packs were readily available it was therefore easier for ward nursing staff to obtain a pre-prepared pack (including the PIPHAS intervention) than it was for them to print out the individual forms from the Trusts intranet.

[See Figure 1 in PIPHAS PDSA Cycles \& Final PIPHAS Form \& Trust Admission Packs Image.]

See supplementary file: ds4375.doc - "The PIPHAS PDSA cycles, the final PIPHAS form and a photograph of our newly created Trust admission packs"

\section{Post-measurement}

Following the implementation of the PIPHAS form, a retrospective review was performed of the inpatient medical notes of the next 100 admissions to Prospect Park Hospital. This looked in particular at their admission clerking documentation which was used to complete our altered post-intervention audit proforma. The audit proforma was amended to allow the documentation of whether or not a PIPHAS form had been completed and uploaded to the patients medical records. As with the baseline measurement, an online audit proforma was used, compiling the data in a secure MySQL database.

An overview of the final audit results are as follows:

A total of 100 patients were included in the post-intervention data collection, of which 50 were males $(50 \%)$. The mean age of the patients was 43.3 years (with a standard deviation of 18.6 years). As with the pre-intervention data collection, the majority of patients were clerked by the on call team (84 out of 100 patients, $84.0 \%$ ). Just over a quarter of patients had a completed PIPHAS form scanned into their medical notes (28 out of 100 patients, $28.0 \%$ ). Of the 100 patients, 75 of them underwent a physical examination on admission to Prospect Park (75.0\%). This was a slight improvement from the pre-intervention data collection (78 out of 111 patients, $70.3 \%)$.

Of the 28 patients with scanned completed PIPHAS forms, all of them underwent a physical examination on admission (28 out of 28 patients, $100 \%$ ) in comparison to those without a scanned PIPHAS form (47 of the 72 patients, $65.3 \%$ ). There was an increase in the documented evidence of the use of a chaperone during examination in those with a scanned PIPHAS form in their notes (nine out of 28 patients, $32.1 \%$ ) in comparison to those without a scanned PIPHAS form (two out of 54 patients, 3.7\%). All of those documenting the use of a chaperone (in both the "with PIPHAS form" and "without PIPHAS form" groups) declared that the chaperone was the same sex as the patient.

In the "with PIPHAS form" group, there was improved documentation of all examination domains within the medical notes when compared to those also undergoing an examination. The subdomains of examination are explored below:

A temperature measurement was recorded in 25 out of 28 patients $(89.3 \%)$ in the "with PIPHAS form" group versus 25 out of 54 (46.3\%) in the "without PIPHAS form" group. A BMI was calculated and recorded in 20 out of 28 patients $(71.4 \%)$ in the "with PIPHAS form" group in comparison to 21 of the 54 patients (38.9\%) in the "without PIPHAS form" group. All 28 out of 28 patients (100\%) in the "with PIPHAS form" group had documented evidence of a full cardiovascular examination, in contrast with the 48 out of 54 patients (88.9\%) in the "without PIPHAS form" group. All of the "with PIPHAS form" group (28 out of 28 patients, $100 \%$ ) had documented evidence of a thorough respiratory examination, compared to 50 out 54 patients (92.6\%) in the "without PIPHAS form" group. Again, all of the "with PIPHAS form" group (28 out of 28 patients, 100\%) had documented evidence of a thorough abdominal examination being performed, whereas only 47 of the 54 patients $(87.0 \%)$ in the "without PIPHAS form" group did. A peripheral neurological examination was recorded in just over half of the "with PIPHAS form 
BMJ Quality Improvement Reports

group" (19 out of 28 patients, $67.9 \%$ ), a much greater number than those in the "without PIPHAS form group" (18 out of 54 patients, $33.3 \%$ ). A central neurological examination (eg cranial nerve testing) was documented as being performed in 19 out of 28 patients (67.9\%) of patients in the "with PIPHAS form" group, in contrast to just 18 of the 54 patients (33.3\%) in the "without PIPHAS form" group.

Unfortunately, there was a marked decrease in the number of patients receiving phlebotomy during their inpatient admission from the pre-intervention (90 out of 111 patients, $81.1 \%$ ) and postintervention studies (46 out of 100 patients, $46.0 \%$ ). But, looking only at the data from the post-intervention data collection, it reveals that slightly more patients in the "with PIPHAS form" group (13 out of 28 patients, $46.4 \%$ ) underwent phlebotomy than in the "without PIPHAS form" group (33 of the 72 patients, $45.8 \%$ ).

As in the pre-intervention baseline measurement, no female patients over the age of 65 years in the post-intervention group were offered or underwent a screening breast examination (zero out of 10 patients, $0 \%$ )

See supplementary file: ds4378.xls - "Appendix - A spreadsheet to show post-intervention raw data and comparison table vs. baseline measurement."

\section{Lessons and limitations}

By carrying out the PIPHAS project a number of lessons have been learnt:

In order to improve the compliance/engagement of fellow physicians in audit interventions it is important that the intervention is needed, well structured, publicised, effective and time-saving from previous methods. In order to improve compliance in subsequent quality improvement projects it is important to publicise an intervention to the intended users before its' distribution.

The process of audit data collection is time consuming. The role of a foundation doctor often involves changing rotations, sometimes even moving Trusts, and this can make data collection logistically challenging. This was especially the case during this quality improvement project, where a "letter of access" was required to gain access to patients' electronic notes in order to carry out the post-intervention data collection. This made the quality improvement project take much longer than was initially anticipated. For future quality improvement projects one must consider the need for IT access and be realistic in setting a target deadline for projects.

The small sample size of five patients during the second PDSA cycle meant that the test run of the intervention did not highlight the need to add a breast examination section to the PIPHAS form (as all of the patients were under the age of 65 years). This meant that compliance with the breast examination audit standard was unlikely to be met. In future quality improvement projects, a larger sample size from a more diverse group of patients should be used, to better reflect the intended intervention population, thereby highlighting potential weaknesses in the intervention.

\section{Conclusion}

The monitoring of the physical health of psychiatric inpatients is as important as the monitoring of their mental health. Research suggests that psychiatric inpatients and those suffering from mental illness are at greater risk of medical co-morbidities, both due to increased prevalence of high risk behaviours (eg smoking) and often as a side-effect of their prescribed medication. It is therefore fundamental, that all psychiatric inpatients receive a prompt and thorough physical examination on admission to hospital to detect any physical ailments that may require intervention or treatment. It is also feasible that the detection of a previously undiagnosed medical complaint (eg diabetes mellitus) may play a significant impact on the pharmacological treatment choice of their underlying mental illness.

The baseline measurement from this audit demonstrates a clear failure to meet audit standards regarding the physical examination of psychiatric inpatients. When reviewing the patient admission clerkings, it was clearly evident that there was no standardised approach to the documentation of physical examination in patients' medical records. By designing and introducing the PIPHAS form, there is a significantly positive trend towards meeting audit standards. This single sided A4 proforma facilitates the rapid documentation of a structured and concise physical examination.

Although their appears to have been relatively poor engagement from colleagues, with only a quarter of the post-intervention group having a scanned PIPHAS form in their notes, the data comparison shows a marked statistical benefit. All those with a completed PIPHAS form scanned into their medical notes had documented evidence of a more thorough examination, covering more examination domains than those without a scanned PIPHAS form. The documentation of temperature, BMI, cardiovascular examination, respiratory examination, abdominal examination, peripheral neurological examination, and central nervous system examination were markedly improved in the "with PIPHAS form" group than the "without PIPHAS form" group. Therefore, one can conclude that the PIPHAS form is a useful tool to improve the speed, efficiency and documentation of a thorough physical examination in new psychiatric inpatients.

\section{References}

1. Carney CP, Jones L, Woolson RF. Medical comorbidity in women and men with schizophrenia: a population-based controlled study. J Gen Intern Med 2006;21(11):1133-7.

2. De Hert M, Correll CU, Bobes J, Cetkovich-Bakmas M, Cohen D, Asai I, Detraux J, Gautam S, Möller HJ, Ndetei DM, Newcomer JW, Uwakwe R, Leucht S. Physical illness in patients with severe mental disorders. I. Prevalence, impact of medications and disparities in health care. World Psychiatry 2011;10(1):52-77.

3. Kisely S, Smith M, Lawrence D, Maaten S. Mortality in individuals who have had psychiatric treatment: population- 


\section{BMJ Quality Improvement Reports}

based study in Nova Scotia. Br J Psychiatry 2005;187:

552-8.

4. Leucht S, Burkard T, Henderson J, Maj M, Sartorius N. Physical illness and schizophrenia: a review of the literature. Acta Psychiatr Scand 2007;116(5):317-33.

5. O'Brien G, Bullock R, Black S, Cormac I, Jones G, Kerr M, Murphy M, Naylor K, Osborn D, Phelan M, Ramsay R. Physical health in mental health; Final report of a scoping group. London: Royal College of Psychiatrists 2009. Royal College of Psychiatrists Occasional Paper OP67.

6. The Royal College of Psychiatrists. Physical Health in Adults.

http://www.rcpsych.ac.uk/healthadvice/improvingphysicalan dmh/physicalhealthinadults.aspx (accessed 31st December 2014).

7. Ustün TB, Rehm J, Chatterji S, Saxena S, Trotter R, Room $\mathrm{R}$, Bickenbach J. Multiple-informant ranking of the disabling effects of different health conditions in 14 countries. WHO/NIH Joint Project CAR Study Group. Lancet 1999;354(9173):111-5.

\section{Declaration of interests}

Nothing to declare.

\section{Acknowledgements}

Mrs Jen Knight (clinical audit manager) - for ongoing support throughout project and facilitating IT "letter of access" to gain access to patients notes for post-intervention data collection when no longer working within the Trust.

Dr Dhaneesha Senaratne, Dr Sanjay Cheema, and Dr Christina Lim (foundation doctors) - for assisting with baseline measurement data collection. 